\title{
LA DEMOCRACIA EPISTÉMICA PUESTA A PRUEBA. RESPUESTA A ROSENKRANTZ Y RÓDENAS
}

\begin{abstract}
I
arlos Rosenkrantz hace un análisis sumamente agudo de la justificación de la democracia sobre la base de su valor epistémico respecto de soluciones moralmente correctas, tal cual la he expuesto en diversos lugares ${ }^{1}$. Creo, sin embargo, que sus críticas no son suficientemente efectivas.
\end{abstract}

1. En primer término, debo comenzar diciendo algo relativamente banal pero que tiene relevancia para fijar cuál debe ser la estrategia teórica correcta al tratar este tema: Aunque pocos fenómenos como la democracia gozan de tan generalizada aceptación -sobre todo en los tiempos de la post-guerra fría-, la justificación moral de la democracia ha demostrado ser una empresa filosófica inmensamente difícil. Todas las concepciones más conocidas naufragan ante los primeros disparos del análisis crítico: las que no están basadas en la transformación de los intereses de la gente (como las concepciones utilitaristas, economicistas, pluralistas y las basadas en la autonomía y el consentimiento) y también las que presuponen una transformación de tales intereses (como las que invocan la soberanía del pueblo o tienen un cariz perfeccionista). Además, casi todas ellas (con excepciones como la de la teoría del consentimiento) se hacen pasibles de una crítica general que es la que no permiten superar la paradoja de la irrelevancia moral del gobierno: cada uno puede determinar que los valores substantivos a los que la justificación apela se satisfacen o se frustran independientemente de las decisiones del gobierno -democrático o no- $\mathrm{y}$, en consecuencia,

${ }^{1}$ En Ética y derechos humanos, Barcelona, 1990 y El constructivismo ético, Madrid, 1990. 
esas decisiones son superfluas para la materialización y determinación de los valores en cuestión.

Por lo tanto, el juego que debemos jugar los filósofos políticos es, según creo, el de proponer la justificación menos expuesta a obvias objeciones, y una vez que se avizora una alternativa con esos méritos tratar de ver hasta dónde resiste, hasta que podemos formular una teoría aún más resistente. La justificación epistémica de la democracia me parece a mí que es por el momento la alternativa teórica menos vulnerable, ya que es la única teoría prima facie plausible que da relevancia moral al gobierno - con tal de que precisamente sea democrático-; parece estar orientada en la dirección correcta de asociar la democracia con la moral (superando el cinismo de las concepciones economicistas y afines de postular que la democracia consiste en una dinámica de composición de intereses en un marco moral fijado en forma elitista); produce esa asociación sólo con la dimensión intersubjetiva de la moral (evitando connotaciones perfeccionistas); y todavía encierra un potencial inexplorado de posibilidades de explicación sobre la conexión entre el procedimiento democrático y ciertas exigencias del discurso moral como la imparcialidad.

Pero Rosenkrantz no eligió jugar este juego en su comentario crítico sino otro: En lugar de explorar todas las posibilidades de una teoría cuyos méritos comparativos ha descrito mejor de lo que podría haberlo hecho yo en las dos primeras secciones de su trabajo (salvo por omitir la incomparable ventaja de permitir explicar la relevancia moral del gobierno democrático), se conforma con encontrar algunas primeras dificultades para rechazarla. Esto pareciera preanunciar que el grueso del trabajo estará dedicado a presentar las bases de una nueva teoría, que absorba todas las ventajas de la que se deja de lado sin presentar las dificultades apuntadas y las más obvias que tienen las más conocidas. Pero no; al final de su trabajo, Rosenkrantz confiesa con su honestidad habitual que no tiene una teoría acabada sino intuiciones bastante firmes; y cuando nos explica hacia donde se orientan sus intuiciones sus someras palabras dejan entrever dos de las concepciones más vapuleadas por la crítica filosófica: la del auto-gobierno (que nunca pudo superar la objeción de que los únicos que se auto-gobiernan son los que integran la mayoría, por lo que se cae o bien en una petición de principio o en una justificación utilitarista); y la que está basada en una concepción del bien republicanista (con todos los peligros perfeccionistas que han sido tantas veces señalados). Por cierto que estas concepciones se ven, además, expuestas a los problemas de las justificaciones «intrínsecas» que destaca tan bien Rosenkrantz: sobre todo el de 
cómo se equilibran los valores que, según ellas, la democracia materializa con los valores que el contenido de las decisiones democráticas pueden violentar.

2. Me parece que Rosenkrantz adoptó esta estrategia en su ofensiva porque, contrariamente a lo que él insinúa, da más importancia a su primera crítica que a la que expone en segundo lugar: o sea cree que la justificación epistémica de la democracia tiene un vicio radical de origen y no meramente el inconveniente de que todavía no ha sido suficientemente bien explicada la relación entre el mecanismo democrático y la exigencia moral de imparcialidad. Ese vicio de origen sería que el objeto primario de su justificación está constituido por procesos políticos ideales y no los que identificamos como democracias reales, y que cuando se proyecta esa justificación sobre la vida política real puede bien legitimar procedimientos de decisión no democráticos - un despotismo ilustrado- y no aquellas democracias reales.

Me da la impresión de que aquí el crítico de la concepción epistémica se confunde. En otro lugar ${ }^{2}$, he sostenido que el concepto de democracia, que solemos emplear en la mayoría de los contextos discursivos, es fundamentalmente una noción normativa, ya que salvo por unos pocos rasgos nucleares -como la libertad de expresión y la votación periódica (insuficientes para identificar los fenómenos denotados por el concepto)-, no es posible determinar qué instituciones son centrales y cuáles son periféricas a la democracia sin una concepción justificatoria que da contenido a ese concepto. Esto ocurre con instituciones como la representación, la división de poderes, el control judicial de constitucionalidad, etc., cuyo carácter inherente o no a la democracia no puede determinarse sin recurrir a una tal concepción justificatoria, con el resultado de que las respuestas son diferentes según se acepte por ejemplo una justificación epistémica o una pluralista. Este carácter del concepto de democracia hace que no se pueda caer en lo que, siguiendo a Sartori ${ }^{3}$, -quien también insinúa que la democracia que es depende de la que debe ser-, podemos llamar «hiper-realismo»: ello consiste en partir de las «democracias reales», con todos sus aspectos tanto prima facie atractivos como viciosos, y buscar concepciones plausibles que las justifiquen directamente. Lo que justifica una concepción que también identifica al objeto de justificación es necesariamente un fenómeno ideal. Aceptar esto

${ }^{2}$ Ver «Conceptions of Democracy and Institutional Design», por aparecer y Filosofía de la Práctica Constitucional, Argentina, también por aparecer.

${ }^{3}$ Ver Democratic Theory, Vol. I, cap. 1, p. 8. Detroit, 1962. 
no es incurrir en un utopismo ilegítimo, y tampoco lo es que no haya ningún fenómeno real que no coincida exactamente con el que resulta plenamente justificado por la concepción normativa en cuestión (ninguna concepción de filosofía política puede satisfacerse completamente en la realidad). Un utopismo ilegítimo consistiría en proveer una concepción que proyectada a la realidad no nos permitiera detectar diferencias de valor entre los fenómenos existentes, a pesar de que intuitivamente percibimos grandes distinciones axiológicas entre ellos. Por ejemplo, una concepción justificatoria de la democracia que parificara en valor cero tanto al régimen de Irak como al de Suecia sería ilegítimamente utópica.

La justificación epistémica no lo es, ya que contiene exigencias que proyectadas a la realidad permiten jerarquizar los procesos políticos existentes según el grado de satisfacción plena a tales exigencias. Las exigencias son las de un proceso de discusión en el que participen, con la mayor libertad igualitaria posible -en términos de tiempo, medios de supresión, oportunidad de conocer los hechos relevantes, etc.- todos los afectados por la decisión, en el que cada uno justifique la solución que propone sobre la base de principios que tengan ciertas propiedades formales, y que expresen qué intereses de ellos o de otros, que deberían ser reconocidos, no lo son por los principios alternativos propuestos por los demás, y un mecanismo de formación de la decisión colectiva que compute igualitariamente la propuesta de cada uno y que haga de ella una función directa de la propuesta con mayor número de adhesiones. Por cierto que no hay, efectivamente, ningún proceso político real que satisfaga plenamente todas estas exigencias, pero es fácil componer una escala de mayor a menor aproximación: podríamos comenzar por un cantón suizo, seguir con las democracias escandinavas (que si bien son indirectas tienen algos grados de participación, acceso igualitario a la comunicación colectiva, sensibilidad frente a los cambios en el consenso colectivo, etc.), continuar aún con la mayor parte de las democracias de Europa Occidental, Australia, Canadá... y bastante más lejos vendrán los Estados Unidos (con baja participación, medios de comunicación privados y selectivos, una excesiva dispersión de las expresiones de la voluntad popular, un sistema rígido de formación del gobierno, control de constitucionalidad contramayoritario, etc.); todavía mucho más lejos aparecen las democracias sudamericanas (con su hiper-presidencialismo y corporativismo), y después México, sin todavía posibilidades reales de alternancia; tal vez al fondo de la fila se avizoren algunos regímenes más bien tendientes al partido único 
y a que los líderes fuertes acostumbren a consensuar sus decisiones en plebiscitos o asambleas populares. Como se ve, en este continuo no aparece para nada un despotismo ilustrado como el que teme Rosenkrantz, ya que, por más méritos que pueda tener en otros sentidos, él carece de una condictio sine qua non de la justificación epistémica de la democracia, que es la participación en el debate y en la decisión de los propios afectados por ella (precisamente por el principio que menciona el crítico de que nadie es mejor juez de sus propios intereses que uno mismo, ya que la imparcialidad de la adopción de los principios propuestos para justificar una decisión se testea verificando si hay intereses de individuos que resultan frustrados sin que haya razones que no se apliquen también a casos en que los intereses de los individuos son satisfechos, estoy convencido de que lo que ha afectado la calidad moral de las decisiones de todos los dictadores argentinos, que en algunos casos pudieron ser bien intencionados, es el de no poderse representar con el peso debido algunos intereses que debieron haber tomado en cuenta).

3. Voy ahora a la segunda crítica: Como advierte bien Rosenkrantz, yo rechazo la visión agregativa de la imparcialidad y las concepciones de la democracia basadas en ella (como la utilitarista, la economicista, la pluralista, y creo que, en el fondo, también las que él ve como más meritorias, ya que deben cuantificar el número de gente que se autogobierna o se perfecciona con la democracia contra quienes se perjudican por las decisiones democráticas). La concepción que defendí en la primera edición de Ética y derechos humanos y después dejé de lado era un poco más complicada que lo que Rosenkrantz describe como visión agregativa de la imparcialidad. Yo no distinguía allí bien el plano ontológico del epistemológico en el que se podía asociar el resultado del discurso moral real (y el de la democracia como regimentación de tal discurso) con principios morales válidos, de ahí es que adaptaba la idea de Rawls y hablaba, como recuerda Rosenkrantz, de la democracia como un caso de justicia procesal pura pero imperfecta. Más que una idea agregativa de la imparcialidad defendía una idea, digamos, graduable de la justicia: si la unanimidad es funcionalmente equivalente -bajo ciertos presupuestos- a la imparcialidad y la justicia se define por la imparcialidad, una solución es más justa que otra, aunque no sea la solución justa, si es aceptada por más gente en ciertas condiciones (de amplitud de participación, debate, etc.). La idea tenía su miga porque no coincide con la visión utilitaria (no se trata de maximizar la satisfacción de los intereses más intensos, sino de cuantificar la aceptación), y recoge 
la intuición de que aún cuando se cometa una injusticia, la injusticia es menor -en igualdad de otras condiciones- sí hay menos gente afectada por ellas.

Pero esto pertenece al pasado. La concepción de la democracia que ahora defiendo es explícitamente epistémica y está basada en la confianza de que el procedimiento democrático -definido como lo propuse sucintamente al comienzo- genera, en grados variables según la medida en que se satisfacen esas exigencias, una dinámica de acción colectiva que tiene una tendencia mayor a aproximarse a decisiones imparciales que cualquier otro procedimiento alternativo de decisión. La dinámica no está sólo generada por la publicidad del debate y la necesidad estratégica de obtener el mayor apoyo posible frente a la eventualidad de que se quiebre cualquier coalición mayoritaria, sino también por la participación de todos en el debate y en la decisión y en la oportunidad que todos tienen de hacer conocer a los demás sus intereses; por la exigencia de que las soluciones que se propugnen se justifiquen (lo que si bien no quiere decir que se justifiquen «válidamente», restringe las posibilidades de defensa de ciertos intereses ya que no cualquier expresión de aprobación de esos intereses cuenta como justificación); por la aplicación en el plano colectivo de las relaciones detectadas por los teoremas de Condorcet y otros; y seguramente por muchos otros mecanismos del sistema democrático -incluso psicológicos y educacionales (aquí puede entrar algo del republicanismo que le atrae a Rosenkrantz) - que intuyo pero no puedo describir todavía con la precisión que querría. Creo que si bien estos rasgos estructurales de la democracia no «garantizan» que los participantes harán un uso público de la razón, inducen a que lo hagan con mayor probabilidad que cualquier otro método de decisión.

Francamente no entiendo por qué dice Rosenkrantz que esta dinámica es «tangencial» a las decisiones moralmente correctas. Ciertamente hay casos individuales en que uno puede estar bastante seguro de que la decisión democrática no es moralmente correcta; pero como cuestión general parece que un procedimiento en el que se den las condiciones antes apuntadas tiende a la larga a producir decisiones más imparciales que las que podría tomar un dictador o un grupo aristocrático (por supuesto siempre que el dictador o aristócrata no sea uno mismo, pero aquí uno tiene que operar con alternativas realistas). Esto es lo que justifica el deber de observar las decisiones democráticas (siempre que el procedimiento se haya aproximado a las condiciones preestablecidas) aún en aquellos casos en que uno está seguro de que ellas son incorrectas; ya que de lo contrario, el procedimiento se socavaría 
y se frustraría nuestro presupuesto de que es, en general, más confiable como método de conocimiento moral.

Las dudas de Rosenkrantz parecen estar fundadas en su creencia de que no hay forma practicable de plasmar algunas de las condiciones que generan la dinámica que tiende hacia la imparcialidad de modo de impedir, por ejemplo, la discriminación recurrente de minorías o el procesamiento de preferencias inmorales o no oponibles a terceros. Dejando de lado esta penúltima referencia a la inmoralidad de las preferencias -que el sistema obviamente no puede excluir a priori sin prejuzgar-, por supuesto que hay mecanismos para materializar aquellas condiciones: No me voy a referir a lo más obvio referido a la libertad de expresión y participación de todas las partes (que tiene, sin embargo, enorme incidencia en la generación de una mayor tendencia hacia la imparcialidad comparada con otros procedimientos) sino a otros aspectos que se intenta que la democracia absorba en grados importantes. Por ejemplo, el rasgo consistente en que, siendo difícil la democracia directa en sociedades complejas, las instituciones mediadoras sean los partidos políticos basados en principios justificatorios o programas y no en intereses crudos como los que representan los grupos corporativos, lo que refleja la exigencia de justificación de los intereses de acuerdo a principios que satisfagan ciertas condiciones formales (este es un rasgo detectado fácticamente aún por los politólogos como Jean Blondel ${ }^{4}$ ); también es relevante que las decisiones centrales se concentren en parlamentos que maximicen la reproducción de las ideologías justificatorias a través de representantes de los partidos y con reglas de debate que procuran reproducir las del discurso moral. Por ejemplo, la existencia de un control de constitucionalidad, orientado a resguardar, como lo propone Ely ${ }^{5}$, la satisfacción de las condiciones del proceso de discusión y decisión democráticas y trate especialmente de impedir que en ese proceso haya minorías marginadas o insulares, como lo decía el juez Stone en la famosa nota 4 de «Carolone Products» ${ }^{6}$. Por ejemplo, el diseño de instituciones, como el parlamentarismo, el multipartidismo y el sistema proporcional acorde que requieran el super-consenso de una democracia «consocional», como lo propone Arend Lijphart ${ }^{7}$, precisamente para los casos

${ }^{4}$ Introducción al estudio comparativo de los gobiernos, trad. Fernando Calleja, Madrid, 1972.

${ }^{5}$ Ver Democracy and Distrust, Cambridge, Mass., 1980.

${ }^{6}$ Ver 304 US 144, pp. 152-153, 1938.

${ }^{7}$ Ver Democracy in Plural Societies, New Haven, 1977. 
que preocupan a Rosenkrantz de minorías que pueden resultar precisamente marginadas por diferencias raciales, lingüísticas, etcétera. Por ejemplo, la descalificación por vía también del control de constitucionalidad de decisiones que no están fundadas en principios morales intersubjetivos sino en concepciones de excelencia humana (que están entre las no oponibles a terceros en la terminología de Rosenkrantz) y respecto a las cuales el método democrático no tiene valor epistémico ya que su validez no está relacionada con la imparcialidad). Todos estos rasgos estructurales que se trata que una democracia satisfaga derivan de la aceptación intuitiva de una concepción epistémica de la democracia, ya que todos ellos están directamente asociados con la idea de imparcialidad que esta concepción maneja.

4. Quiero terminar diciendo algo acerca de tal concepción de la imparcialidad. Creo que Rosenkrantz la describe erróneamente como una armonización de intereses sobre la base de principios. El error está en no advertir que la imparcialidad no consiste en subsumir intereses en principios (que pueden ser muy parciales), sino que califica a los principios mismos, o mejor dicho, a su adopción. Es por ello que la imparcialidad es una exigencia procedimental del discurso moral, conduciendo a la adopción de principios como resultado de la práctica de ese discurso. Los contornos completos de la idea de imparcialidad deben ser todavía elucidados, pero creo que comprenden las ideas más básicas, en orden creciente de complejidad, de generalidad -el que los principios no debe distinguir situaciones sobre la base de nombres propios o descripciones definidas-; de universalidad -el que los principios deben aplicarse a todas las situaciones que no puedan distinguirse sobre la base de propiedades relevantes para los mismos principios-; de separabilidad e independencia de las personas, como advierte bien el propio Rosenkrantz; tal vez incluso la idea de que los procesos naturales deben afectar a todos los miembros del grupo relevante y no sólo a aquellos sobre los que recaen físicamente. Este conjunto de ideas, y seguramente otras que se me escapan, que integran la de imparcialidad sirven en el discurso moral de criterios reguladores para la adopción de principios sobre el tratamiento armónico de los intereses de la gente; y el ejercicio mismo de la práctica del discurso moral $-\mathrm{y}$, en menor grado, el de su versión institucionalizada que constituye la democracia- va induciendo a los partícipes a aplicar tales criterios en la selección de principios.

Para decir lo mismo en los términos de preferencias que emplea Rosenkrantz: la imparcialidad es un calificativo de las preferencias impersonales de segundo nivel que se refieren al 
tratamiento de las preferencias personales (que versan sobre la propia vida y el carácter de un individuo). La democracia epistémica es una contienda sólo de preferencias impersonales -que son las únicas a las que se pueden aplicar los criterios de imparcialidad-; de ahí la necesidad de justificación de los intereses y la exclusión de actitudes perfeccionistas. Esto no quiere decir que en el debate democrático no se pueden y aún deban exponer intereses o preferencias personales; al contrario, deben exponerse siempre que se los justifique, ya que ellas son el objeto de referencia de los principios o preferencias impersonales y su ignorancia no justificada es el test de la parcialidad de estas últimas. La aceptación por parte de los presuntos interesados es, a su vez, el signo de que no se da tal ignorancia, y es, por eso que, a mayor aceptación -bajo las condiciones apuntadas- se da efectivamente una mayor presunción de la validez moral de la decisión.

Por cierto que hay todavía muchos puntos oscuros acerca de la relación entre imparcialidad -y, en consecuencia, la moral intersubjetiva- y democracia. Pero tales puntos oscuros constituyen desafíos para fortalecer la justificación epistémica y no signos de que ella está herida de muerte. No es fácil encontrar otra teoría que ofrezca razones para hacer moralmente relevante al gobierno democrático, sin identificar sus decisiones con las moralmente correctas, ocupando una posición intermedia entre tal convencionalismo ético y un elitismo epistémico, sin necesitar hacer un balance entre los valores materializados por el método democrático o sus consecuencias y otros valores que puedan estar violentados por el contenido substantivo de sus decisiones o sus consecuencias, y permitiendo justificar los límites a la democracia -por violación de sus procedimientos, por incursionar en ideales de virtud personal, etc.- sobre la base de las mismas razones que la justifican. Creo que todavía vale la pena hacer un esfuerzo para profundizar todas las posibilidades de esta justificación antes de abandonarla por una mejor, y espero que Carlos Rosenkrantz siga contribuyendo con su talento a esclarecer tanto sus aspectos más promisorios como los más dudosos.

\section{II}

En cuanto al comentario de Angeles Ródenas, debo decir, en primer lugar, que hace un análisis muy perceptivo de cómo fue evolucionando mi visión de la democracia a través del tiempo. En efecto, abandoné la idea de que la democracia es una forma de 
justicia procesal pura pero imperfecta cuando advertí que ello era una modalidad de lo que ahora llamo "constructivismo ontológico», que presenta una serie de dificultades a mi juicio insuperables. La posición que adopté últimamente, el «constructivismo epistemológico», permite distinguir entre la función constitutiva de la validez de los principios morales que cumplen los presupuestos de la práctica del discurso moral de la función cognitiva que cumple el consenso que se alcanza en la culminación de instancias de ese discurso. A su vez, el proceso democrático de decisión colectiva, al reemplazar la exigencia de consenso unánime de ese discurso por la decisión mayoritaria en un momento prefijado preserva parte del valor epistémico de aquel consenso, gracias a una serie de factores subyacentes a ese proceso que determinan una dinámica de interacción que tiende a la imparcialidad. Sin embargo, la posibilidad de acceso al conocimiento moral en cuestiones intersubjetivas no es exclusiva del proceso colectivo de discusión y decisión democráticas, sino que también puede predicarse, aunque en un grado considerablemente menor de confiabilidad, de la reflexión individual, lo que legitima el aporte que cada uno puede hacer a ese proceso colectivo.

Lo que digo en réplica a la crítica de Rosenkrantz sobre la capacidad de este modelo de permitir legitimar democracias reales se aplica también a las observaciones similares de Ródenas. Si el modelo permite, como ella reconoce, derivar prescripciones para la introducción de democracias más perfeccionadas, debe permitir establecer qué cerca o qué lejos estarán las democracias reales de aquellas democracias más próximas al modelo ideal. La legitimidad de tales democracias reales no es una cuestión de todo-o-nada sino de grado.

En primer lugar, habrá democracias reales, más legítimas que otras, y todas ellas seguramente serán más legítimas, de acuerdo a la justificación propuesta, que estructuras no-democráticas de decisión colectiva. En segundo lugar, lo que importa realmente establecer cuando se discute la legitimidad de una democracia real es si tiene el grado de legitimidad suficiente como para fundamentar nuestro deber moral de basar nuestras acciones o decisiones en las conclusiones, no de nuestra reflexión individual, sino del proceso colectivo de decisión y discusión. En la mayoría de los casos de democracias reales la respuesta debe ser afirmativa, ya que, por imperfectos que sean los procedimientos de discusión Y decisión que en ellas se desarrollan, parecen ser más conducentes a orientarse, en general y a la larga, hacia soluciones que contemplen imparcialmente los intereses de los afectados, que la reflexión individual y aislada de cualquiera de nosotros. 
Por cierto, que la segunda función de mi modelo que señala Ródenas también tiene suma importancia: la de servir de punto de referencia para posibles reformas de los sistemas democráticos existentes para expandir su capacidad epistémica. Pero esta segunda función no sólo se da interconectada con la anterior sino que, a veces, entra en cierta tensión con ella. En primer término, si la democracia existente tiene la mínima capacidad epistémica como para justificar que actuemos según sus determinaciones y no las que provienen de nuestra reflexión individual, es a través de esos procedimientos democráticos reales que debe propugnarse su perfeccionamiento. No cabe, como muchos adeptos a un elitismo epistémico en materia moral lo propugnan (por ejemplo la guerrilla de los años 70) recurrir a métodos antidemocráticos para establecer la «verdadera» democracia. Segundo, hay veces que es necesario desconocer alguna decisión democrática como forma de promover el perfeccionamiento del proceso democrático, como deben hacerlo, por ejemplo los jueces a través del control judicial de constitucionalidad (por ejemplo, en el famoso fallo de la Corte Suprema de los Estados Unidos en Brown vs. Board of Education, en el que se desconocieron decisiones democráticas de los estados favorables a la segregación racial en materia educativa, tomando en cuenta, o debiendo tomar en cuenta -ya que la Corte no es suficientemente explícita sobre el punto- que la segregación educativa afecta a la calidad epistémica del proceso democrático al no permitir la confraternización necesaria para la adopción de soluciones imparciales). 